\title{
Nonlinear barotropic tides and bores in estuaries
}

\author{
By ANDREAS MÜNCHOW and RICHARD W. GARVINE, College of Marine Studies, University of \\ Delaware, Newark, DE 19716, USA
}

(Manuscript received 18 April 1989; in final form 11 October 1990)

\begin{abstract}
The propagation of tides from the coastal ocean into shallow estuarine waters often produces characteristic nonlinearly induced asymmetries of velocity and water level in time series. Until recently (Aubrey and Speer, 1985) a sound physical understanding of related phenomena has been difficult to obtain as flow field data are rare. Numerical studies usually model a single estuary only; the modeled parameter space is often limited. From the vertically averaged governing equations that include nonlinear terms and bottom friction, we deduce that three dimensionless parameters set the character of the barotropic tidal response for channel shaped estuaries. We then develop a time-dependent numerical model based on the method of Lax and Wendroff. We use the model to explore the response over a wide range of the three parameters. Tidal responses include damped waves, hydraulic regimes, and tidal wave breaking or bore development. Where field data are available in the case of the Conwy Estuary, UK, water level and velocity compare favorably with model prediction.
\end{abstract}

\section{Introduction}

The propagation of tides from the coastal ocean into shallow estuarine waters often produces characteristic nonlinearily induced asymmetries of velocity and water level. Hansen (1956) first gave numerical solutions of nonlinear tidal propagation for the Ems Estuary, Germany. Kreiss (1957) studied the ebb-flood asymmetry of the tidal flow field analytically with perturbation methods. $\mathrm{He}$ attributed the distortion of the tidal wave to quadratic friction acting in concert with nonlinear advection. Breaking of the tidal wave has been observed in several estuaries: the Severn and Trent in England (Cornish, 1934 and Rowbotham, 1983), the Hugli in India (Masumder et al., 1984), the Tsien Tang in China (Cornish, 1934), and in the Shubenacadie in Canada. Abbott (1956) investigated wave breaking analytically and deduced that nonlinear advection was the main cause of bore development. Prandle (1985) studied the dynamical effects of exponentially varying channel geometries with a linear tidal model. All these early studies employed specific, highly simplified estuarine dynamics in order to address particular physical processes.

LeBlond (1978) introduced a new physical inter- pretation of highly nonlinear and frictionally dominated tidal flows. Traditionally, tides are conceived as long waves with energy propagating both into and out of a forced basin. In order to explain large phase lags in low water in the St. Lawrence Estuary, Canada, LeBlond (1978), instead, postulated that friction dissipates the tidal energy completely during flood. In this concept, the tidal energy propagates only landward, similar to the conduction of heat away from a source.

In Section 2 of this paper, we formulate a barotropic (vertically averaged) model based on the governing equations of momentum and mass continuity. Using scaling analysis we find that three dimensionless parameters set the character of the tidal response. In Section 3, we introduce a numerical scheme to solve these equations for variable channels. We utilize work of Houghton and Kasahara (1968) and O'Donnell and Garvine (1983) who applied the numerical one-step scheme of Lax and Wendroff (1960) to highly nonlinear but inviscid problems simulating air flow over ridges and buoyant plumes, respectively. In Section 4 we present water level and velocity data from the Conwy Estuary, UK, and compare them with our model predictions. The Conwy Estuary serves as a particular example of strongly non- 
linear tidal dynamics. In Section 5 we explore the parameter space of barotropic tides in shallow estuaries. This section is a generalization of the work of LeBlond (1978) and Speer and Aubrey (1985). Section 6 concludes the paper.

\section{Tidal model formulation}

We assume estuaries that are channel-shaped and narrow enough to ignore Coriolis force, i.e., the barotropic Rossby radius of deformation is much larger than the width of the estuary. We also neglect transverse flow components. The mathematical representations of cross-sectional averaged tidal dynamics for an incompressible fluid are:

$\frac{\partial u^{*}}{\partial t^{*}}+\frac{1}{2} \frac{\partial u^{* 2}}{\partial x^{*}}+g \frac{\partial \eta_{\mathrm{a}}^{*}}{\partial x^{*}}+\frac{\tau_{\mathrm{b}}^{*}}{\rho^{*}} \frac{p^{*}}{A^{*}}=0$,

$b^{*} \frac{\partial \eta^{*}}{\partial t^{*}}+\frac{\partial A^{*} u^{*}}{\partial x^{*}}=0$.

Here asteriks denote dimensional variables; $u^{*}\left(x^{*}, t^{*}\right)$ and $\eta_{\mathrm{a}}^{*}\left(x^{*}, t^{*}\right)$ are the dependent variables, axial velocity and water level, respectively; $\left(x^{*}, t^{*}\right)$ are axial distance from the mouth and time; $b^{*}$ represents width; $p^{*}\left(x^{*}, t^{*}\right)$ is the wetted perimeter; and $A^{*}\left(x^{*}, t^{*}\right)$ is the wet crosssectional area. Bottom and side wall stresses $\tau_{\mathrm{b}}^{*}$ are represented as

$\tau_{\mathrm{b}}^{*}=C_{\mathrm{D}} \rho^{*} u^{*}\left|u^{*}\right|$,

where $\rho^{*}$ is the reference density and $C_{\mathrm{D}}$ a frictional drag coefficient. We neglect frictional stresses at the free surface.

To incorporate the nonlinear effects which arise from substantial changes of $A^{*}$ with time, we model the cross-sections as shown in Fig. 1. Motivated by observed shapes of cross-sections in natural channels, we assume a trapezoidal crosssection above a shallow, rectangular ebb channel of depth $h_{0}^{*}$, always filled with water. The top of the ebb channel constitutes a reference for the tidal water level elevations which, then, is always positive. In constant depth channels $\eta^{*}=0$ is an absolute reference, but in Section 4 we model an estuary with an axially sloping river bed and $\eta^{*}=0$, then, is no longer an absolute reference level. Thus, the vertical distance from the top of the ebb channel to an absolute datum constitutes another geometric input function, $c^{*}\left(x^{*}\right)$ with $\eta_{\mathrm{a}}^{*}=\eta^{*}+c^{*}$. However, the variable estuarine width is

$$
\begin{aligned}
& b^{*}\left(x^{*}, \eta^{*}\left(x^{*}, t^{*}\right)\right) \\
& \quad=b_{0}^{*}\left(x^{*}\right)+a^{*}\left(x^{*}\right) \eta^{*}\left(x^{*}, t^{*}\right),
\end{aligned}
$$

where $b_{0}^{*}$ and $a^{*}=\delta b_{1}^{*} / \delta \eta_{1}^{*}+\delta b_{2}^{*} / \delta \eta_{2}^{*}$ are known geometric input functions representing axial width variation and the constant slopes of the side walls, respectively. Thus, the model includes the effects of variable width and cross-sectional area.

Since the structure of the governing equations is second order in $x^{*}$ or $t^{*}$, two boundary conditions must be applied. Here, specification of the freshwater flow at the head and water level at the mouth of the estuary as functions of time suffice.

Next, we scale the equations by introducing the dimensionless variables

$t \equiv t^{*} / T, \quad p \equiv p^{*} / B, \quad b \equiv b^{*} / B$,

$A \equiv A^{*} / H B, \quad \eta^{*} \equiv \eta / \eta_{0}, \quad \eta_{\mathrm{a}}^{*} \equiv \eta_{\mathrm{a}} / \eta_{0}$,

$b_{0} \equiv b_{0}^{*} / B, \quad h_{0} \equiv h_{0}^{*} / H, \quad a \equiv a^{*} \eta_{0} / B$,

where $T, B, H$, and $\eta_{0}$ are tidal period, mean width, mean depth, and water level amplitude at the estuarine mouth, respectively. These scales are known a priori for any particular estuary and are independent of the particular dynamics. In contrast, the scales $U$, for velocity, and $L$, for length,

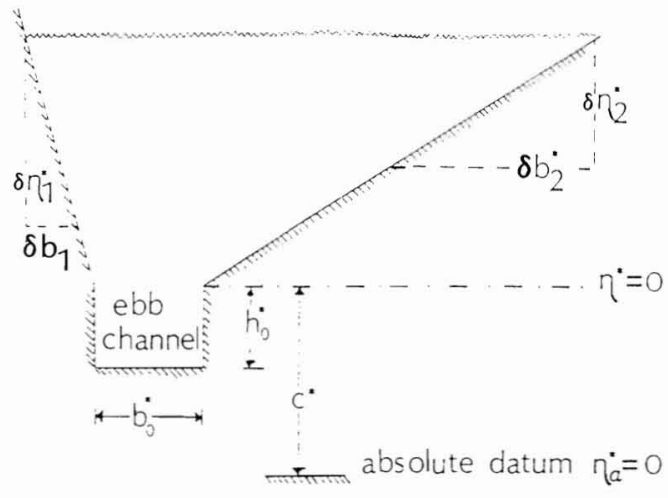

Fig. 1. Representation of variable cross-sectional area in the Lax-Wendroff model. Low water never falls below $\eta^{*}=0$. 
depend on the dynamics and are used to define scaled variables as follows:

$u \equiv u^{*} / U, \quad x \equiv x^{*} / L$.

$U$ and $L$ depend on the tidal response of the estuary and will be found later in terms of the independent scales $T, H, \eta_{0}$, and the drag coefficient $C_{\text {D. }}$.

The scaled continuity equation is now:

$\varepsilon L b /(U T) \partial_{t} \eta+\partial_{x}(A u)=0$,

where $\varepsilon \equiv \eta_{0} / H$. In order to simplify the notation we represent partial derivatives by subscripts. A necessary condition for a balance in (5) requires that both terms are of order one, i.e., $U \equiv \varepsilon L / T$. Then the scaled momentum equation is

$$
\begin{aligned}
& \left(L / L_{\lambda}\right)^{2} \partial_{t} u+\frac{1}{2} \varepsilon\left(L / L_{\lambda}\right)^{2} \partial_{x} u^{2} \\
& \quad+\partial_{x} \eta+\left(L / L_{\mathrm{f}}\right)^{3} u|u| p / A=0,
\end{aligned}
$$

where $L_{\mathrm{f}} \equiv\left[g H^{2} T^{2} /\left(\varepsilon C_{\mathrm{D}}\right)\right]^{1 / 3}$ and $L_{\lambda} \equiv \sqrt{g H} T$ are the frictional length scale introduced by LeBlond (1978) and the tidal wavelength, respectively. The effective length scale, $L$, for a particular dynamic regime may be any one of three candidates:

(a) $L_{g}$, the geometric length scale related to estuarine bathymetry, for example, the distance from the mouth to the head of the estuary; this scale does not appear explicitly in eq. (6), but enters through the boundary condition at the head;

(b) $L_{\lambda}$, the tidal wavelength;

(c) $L_{f}$, the frictional length scale.

In all following computations of velocity and water level we used $L=\min \left(L_{\mathrm{f}}, L_{\lambda}, L_{\mathrm{g}}\right)$.

Three independent dimensionless parameters $(\varepsilon, \phi, \gamma)$ can now be defined from the above scalings:

$\varepsilon \equiv \eta_{0} / H, \quad \phi \equiv L_{\mathrm{f}} / L_{\lambda}, \quad \gamma \equiv L_{\mathrm{g}} / L_{\lambda}$.

The parameter $\varepsilon$ depends only on known scales and indicates the importance of the nonlinearities because it appears in the advective and frictional terms. The two length scale ratios, $\phi$ and $\gamma$, characterize the response of the estuary, since their magnitudes select the term which balances the surface pressure gradient in eq. (6). We thus find that the barotropic tidal response of shallow estuaries depends upon three dimensionless parameters. In Section 5 we explore the model tidal response within the space defined by these three parameters.

\section{Solution technique}

The scheme of Lax and Wendroff (1960) solves the governing equations numerically. It requires equations written in conservation law form, such as

$\partial_{t} P+\partial_{x} Q+R=0$

where $P, Q$, and $R$ are vectors such that $Q=Q(P)$ and $R=R(P)$. The dimensionless momentum and continuity equations above transform into this form when $\zeta(\eta)=b_{0} \eta+\frac{1}{2} a \eta^{2}$ is used as a dependent variable representing wetted crosssectional area above the top of the ebb channel (Fig. 1). The form of eq. (8) is then obtained from eqs. (5) and (6) with

$P=\{u, \zeta\}$,

$Q=\left\{\varepsilon \min (1, \phi, \gamma)^{2} u^{2} / 2+\eta, A u\right\}$,

$R=[\min (1, \phi, \gamma) / \phi]^{3}\{u|u| p / A, 0\}$,

where

$A=h_{0} b_{0}+\varepsilon\left(b_{0}+\frac{1}{2} a \eta\right) \eta$,

$\eta(\zeta)=-b_{0} / a+\left(b_{0}^{2} / a^{2}+2 \zeta / a\right)^{1 / 2}$.

The finite difference scheme of Lax and Wendroff (1960) emerges from a Taylor series expansion in time of the dependent variable $P$ carried through second order in $(\delta t)^{2}$, where $\delta t$ is the time step. Expressing the time derivatives by spatial derivatives gives

$$
\begin{aligned}
& P(t+\delta t)=P(t)-\left(\partial_{x} Q+R\right) \delta t+\left\{\partial_{x}\left[J\left(\partial_{x} Q+R\right)\right]\right. \\
& \left.+G\left[\partial_{x} Q+R\right]\right\} \frac{1}{2} \delta t^{2}+\mathrm{O}\left(\delta t^{3}\right),
\end{aligned}
$$

where $J$ and $G$ are the Jacobian matrices:

$J=\partial_{P_{j}} Q_{i}=\left\{\begin{array}{cc}\varepsilon \min (1, \phi, \gamma)^{2} u & \partial_{\zeta} \eta \\ A & u \partial_{\zeta} A\end{array}\right\}$, 


$$
\begin{aligned}
G & =\partial_{P_{j}} R_{i}=[\min (1, \phi, \gamma) / \phi]^{3} \\
& \times\left\{\begin{array}{cc}
2|u| p / A & u|u|\left(A^{-1} \partial_{\zeta} p+p \partial_{\zeta} A^{-1}\right) \\
0 & 0
\end{array}\right\} .
\end{aligned}
$$

Eq. (9) can be written in finite difference form (Houghton and Kasahara, 1968) as:

$$
\begin{aligned}
& P(t+\delta t)=P(t)-\left(\delta_{i} Q+R_{i}\right) \delta t \\
& \quad+\left\{\left[J^{+}\left(\delta_{i+1 / 2} Q+R^{+}\right)\right.\right. \\
& \left.\quad-J^{-}\left(\delta_{i-1 / 2} Q+R^{-}\right)\right] / \delta x \\
& \quad+\left[G\left(\delta_{i} Q+R_{i}\right]\right\} \delta t^{2} / 2,
\end{aligned}
$$

where

$\delta_{i} \psi \equiv \frac{1}{2}\left(\psi_{i+1}-\psi_{i-1}\right) \delta x$,

$\delta_{i+1 / 2} \psi \equiv\left(\psi_{i+1}-\psi_{i}\right) / \delta x$,

$\delta_{i-1 / 2} \psi \equiv\left(\psi_{i}-\psi_{i-1}\right) / \delta x$,

$\psi^{+} \equiv \frac{1}{2}\left(\psi_{i+1}+\psi_{i}\right)$,

$\psi^{-} \equiv \frac{1}{2}\left(\psi_{i}+\psi_{i-1}\right)$,

and $\psi$ represents an operand.

Lax and Wendroff (1960) showed that a necessary condition for the stability of the numerical scheme is the Courant-Friedrich-Lewy condition

$N_{\mathrm{C}} \equiv\left[u_{\max }+(A / b)_{\max }^{1 / 2} / \min (1, \phi, \gamma)\right] \delta t / \delta x<1$,

where $u_{\max }$ is the magnitude of the maximum particle velocity, $[A / b)_{\max }^{1 / 2} / \min (1, \phi, \gamma)$ is the maximum tidal phase velocity in the modeled domain, and $N_{\mathrm{C}}$ is the Courant number. Muenchow (1988) verified the numerical scheme against analytical solutions of linear tidal and nonlinear, but inviscid, bore propagation in constant breadth and depth channels. The latter problem models the flow evolving after the breaking of a dam (Stoker, 1947).

Lax and Wendroff (1960) designed their numerical scheme so that it conserves mass and momentum across discontinuities. Therefore, the LaxWendroff scheme, as compared with others, remains stable even when discontinuities, such as bores, arise unexpectedly in the modeled domain. It is accurate to order $(\delta t)^{2}$, but once a bore has formed, the flow variables behind the bore oscillate slightly. The speed of propagation of the bore is accurate only within 10 percent, as compared with analytically derived propagation speeds (Richtmayer and Morton, 1967). Here, we focus attention on the distortion of the tidal wave as it enters shallow estuaries rather than on the details of bores themselves. Thus, the modest errors associated with the Lax-Wendroff model when bores occur are acceptable. The next section illustrates that the Lax-Wendroff model can be applied to highly nonlinear tidal dynamics of a real estuary which exhibits large variations of estuarine width and depth.

\section{Application to the Conwy estuary}

Fig. 2a shows a map of the Conwy Estuary, UK, from its mouth at Deganwy to its tidal limit at Llanwrst. The estuary has tidal flats, is shallow in depth, and has a vertical salinity structure that is partially to well mixed. Dramatic changes in estuarine width are seen in Fig. $2 b$ depicting two typical cross-sections along with mean low and high water levels at Conwy. On the tidal time scale, the wetted cross-sectional area varies by an order of magnitude with time and position along the estuary. Wallis and Knight (1984), West and Mangat (1986), and Shiono and West (1987) studied the velocity field of the Conwy Estuary intensely in order to calibrate numerical models, determine transport processes, and resolve bottom generated turbulence.

Fig. 3 shows time series of observed water level and velocity at two stations obtained by the senior author in June 1986. The data were band-pass filtered with a Lanczos filter ( 0.5 and $25 \mathrm{~h}$ cut-off periods). Near the mouth at Conwy the water level (Fig. 3a) exhibits a nearly harmonic wave form, whereas at Dolgarrog the wave crests are steep and sharp (Fig. 3b), i.e., a nonlinearly distorted wave results as the tide propagates into the estuary. Further nonlinearity appears in the velocity time series, as the flood currents last for only 3 to $4 \mathrm{~h}$ (Figs. 3c, d). Strong asymmetry in the tidal signal is clear.

The objective of this section is to apply the model to the Conwy Estuary and, in particular, to explain the observed nonlinearities. The only adjustable parameter with which to tune the model 


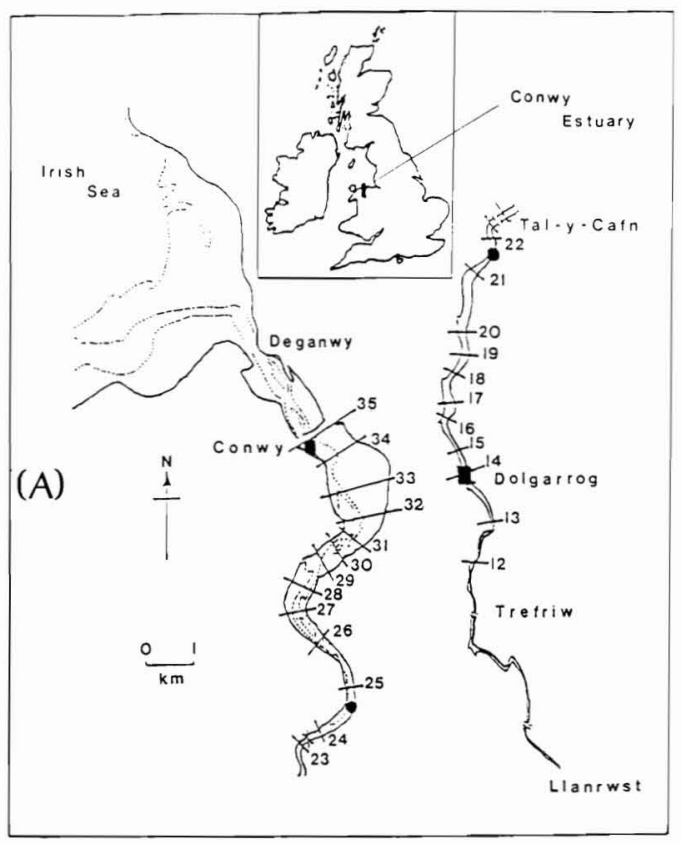

is the frictional drag coefficient, $C_{\mathrm{D}}$. We varied the coefficient to best reproduce the observed tidal water level at Dolgarrog and adopted $C_{\mathrm{D}}=10^{-2}$. The total model domain extends from Conwy to Llanwrst and consists of 35 equally spaced spatial grid points. For the Conwy Estuary $(H=3 \mathrm{~m})$, then, the length scales defined in the previous section are:

$L_{\mathrm{g}} \simeq 21 \mathrm{~km}, \quad L_{\mathrm{f}} \simeq 34 \mathrm{~km}, \quad L_{i} \simeq 320 \mathrm{~km}$.

Therefore, the dimensionless parameters are $\varepsilon \equiv$
(B)

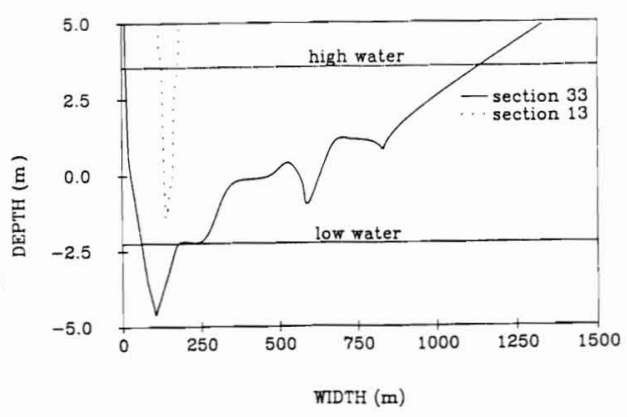

Fig. 2. (a) Map of the Conwy Estuary, UK. The map on the left continues at the right. The numbers indicate stations for bathymetric data. Locations of the tide gauges ( $)$ are Conwy and Dolgarrog. Positions of Aanderaa current meter moorings $(\bullet)$ are near Sections 22 and 25 . The dashed line marks areas which are dry at lower water. (b) Two profiles of contrasting crosssections (see (a) for the locations). Note the dramatic variations of cross-sectional area along the channel and with time, i.e., compare wetted areas at times of high and low water.

$\eta_{0} / H=0.8, \quad \phi \equiv L_{\mathrm{f}} / L_{\lambda}=0.11, \quad$ and $\gamma \equiv L_{\mathrm{g}} / L_{\lambda}=$ 0.07 . Imposed boundary conditions are water level at Conwy (Fig. 3) and freshwater velocity at the head (Llanwrst).

The model predicts water level and velocity which we compare in Fig. 4 with water level at Dolgarrog and velocity data at two sections (see Fig. 2 for the locations). The phase of water level data at Dolgarrog lags the predicted phase by about 0.5 hours (Fig. 4a). The model overestimates the low water levels, but the water depth at this stage of the tidal cycle is extremely shallow

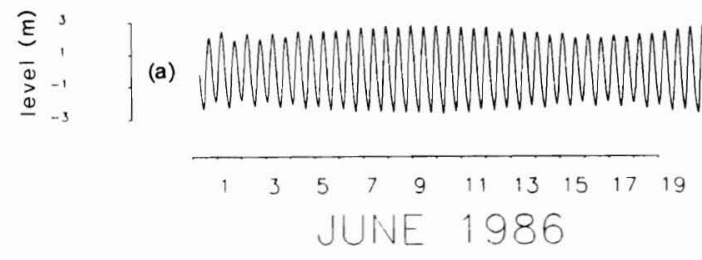

SECTION 22

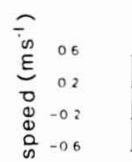

Fig. 3. Time series of water level at (a) Conwy and (b) Dolgarrog (note the increase of nonlinear properties); velocity near (c) Section 22 and (d) Section 25.

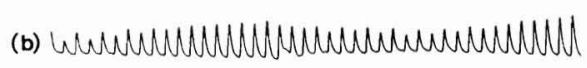

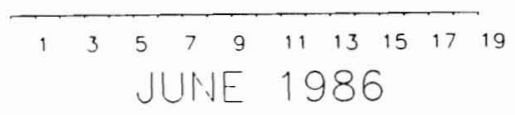

SECTION 25

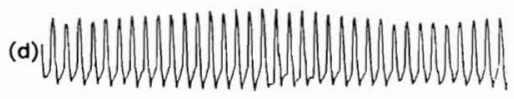


(a)

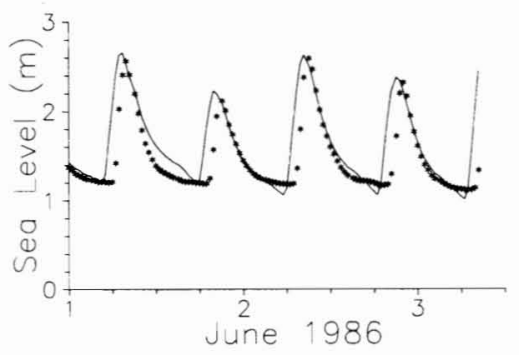

(b)

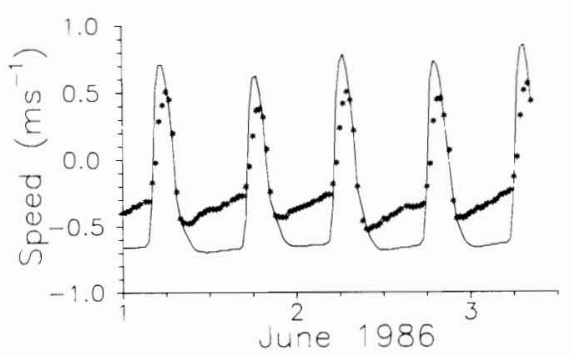

(C)

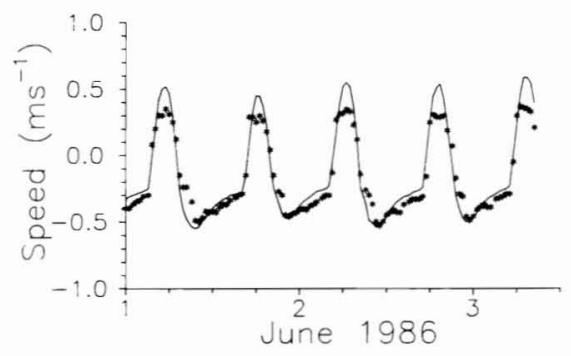

Fig. 4. Comparison of model predictions for the Conwy Estuary with data for five tidal cycles: (a) Water level at Dolgarrog (Section 14), (b) velocity near Section 22, (c) velocity near Section 25 . The dots indicate the data and the solid lines represent model predictions (see Fig. 2a for the locations and Fig. 3 for the data).

$(<0.5 \mathrm{~m})$. Figs. $4 \mathrm{~b}$ and c compare velocity data with predictions. Since the velocity was measured at only a single point in the cross-section while the model predicts cross-sectional averaged velocities, we can only compare the velocity phase quantitatively, because the phase varies little with width and depth. The model simulates the phase and even the amplitude of the velocity data very well. The highly distorted, asymmetric waveform representing only 3 to $4 \mathrm{~h}$ of flood currents is in excellent agreement with the data.

Fig. 5 shows a typical momentum balance at two grid points for a single tidal cycle. Close to the mouth (Fig. 5a) the acceleration term, $\partial, u$, con- (a)

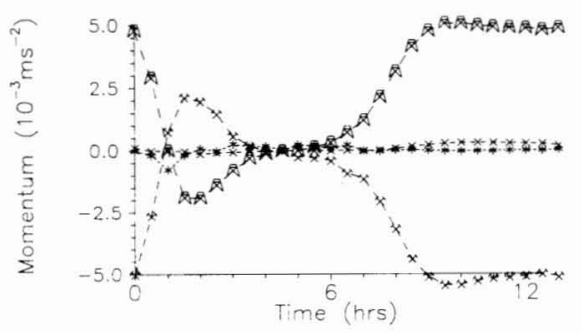

(b)

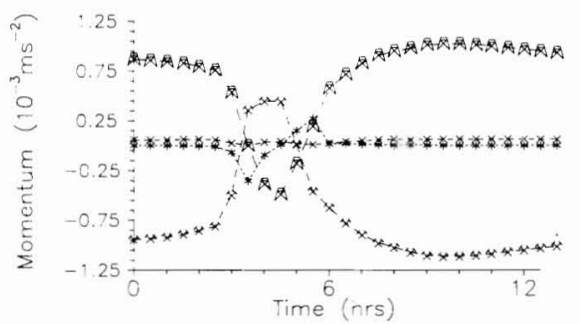

Fig. 5. Model momentum balance for the Conwy Estuary (a) near the mouth and (b) near the head of the estuary for one tidal cycle. Negative friction corresponds to flood currents. Note that the advection and acceleration terms are small throughout the tidal cycle at both positions.

tributes only near slack water, is negligible most of the time, and is zero when averaged over $T$. The wave form is nearly symmetric near the mouth (Fig. 5a), but becomes asymmetric further landward (Fig. 5b). The advection term, $\frac{1}{2} \partial_{x} u^{2}$, is almost negligible except near slack water when velocities and the resulting friction are small. Otherwise, the balance is between the surface pressure gradient, $\partial_{x} \eta$, and friction. The time averaged momentum balance is between the friction and slope terms (hydaulic balance) which dominate the balance throughout the estuary. However, the free surface slope changes within an hour from its ebb to maximum flood value.

\section{Numerical experiments}

Table 1 summarizes the experiments conducted to explore the parameter space $(\varepsilon, \phi, \gamma)$. Barotropic tides in channels of constant breadth and depth with no riverine inflow will be classified and inter- 
Table 1. Summary of numerical experiments

\begin{tabular}{|c|c|c|c|c|}
\hline \multirow[t]{2}{*}{ Experiment } & \multicolumn{3}{|c|}{ Parameters } & \multirow[t]{2}{*}{ Comments } \\
\hline & $\varepsilon$ & $\phi$ & $\gamma$ & \\
\hline case 1: & 0.8 & 0.25 & 0.10 & bore on flood \\
\hline case 2: & 0.8 & 0.10 & 0.10 & hydraulic case \\
\hline case 3 : & 0.4 & 0.10 & 0.10 & marginal hydraulic \\
\hline case 4 : & 0.4 & 0.25 & 0.10 & damped wave \\
\hline case $5:$ & 0.4 & 0.50 & 0.10 & damped wave \\
\hline case 6 : & 0.4 & 0.25 & 0.50 & marginal bore \\
\hline case 7: & 0.4 & 0.50 & 0.50 & bore on flood \\
\hline case 8 : & 0.8 & 0.50 & 0.50 & flood and ebb bore \\
\hline Severn: & 0.8 & 0.15 & 0.14 & bore on flood \\
\hline Conwy: & 0.8 & 0.11 & 0.07 & hydraulic regime \\
\hline
\end{tabular}

preted in terms of these parameters. Next, we discuss typical cases to illustrate the variety of estuaries the model can address.

\subsection{Highly nonlinear flows: $\varepsilon=0.8$}

When $\varepsilon=0.8$, we expect highly nonlinear responses. Bores develop in case $1 \quad(\varepsilon=0.8$, $\phi=0.25, \gamma=0.1)$. Figs. $6 \mathrm{a}$ and $\mathrm{b}$ give the velocity and water level contours in the $(x, t)$ plane for one tidal cycle. Solid and broken lines representing ebb and flood currents, respectively. During ebb the currents vary mainly with axial position, not time, whereas during flood the currents vary dominantly with time. This ebb-flood asymmetry in velocity is most evident near the estuarine head where the ebb phase lasts about two-thirds of a cycle. In Fig. 6b solid and broken contour lines represent elevations above and below the mean water level, respectively. Finally, for the same tidal cycle Fig. 6c depicts two families of characteristic curves. They form, mathematically, a curvi-linear coordinate system on which the Riemann invariants $u^{*} \pm\left[g\left(A^{*} / b^{*}\right)\right]^{1 / 2}$ stay constant. Physically, the upward sloping characteristics in Fig. $6 \mathrm{c}$ correspond to long gravity waves which propagate from the head of the estuary toward its mouth, whereas the downward sloping characteristics represent waves that propagate in the opposite direction. We interpret converging characteristics in the figure as compressional waves (Stoker, 1948) which have coalesced to form a bore. The envelope of these coalescing characteristics thus tracks the bore path in $(x, t)$ space. As seen in Fig. 6c for case 1, a tidal bore forms near the estuarine mouth and propagates upstream with a
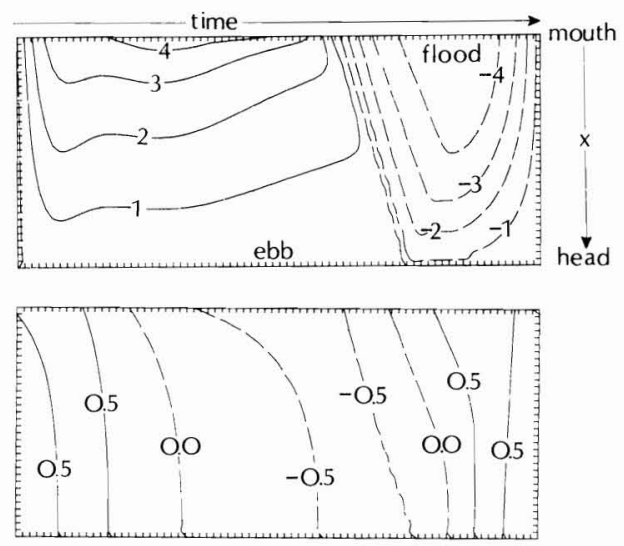

supercritical

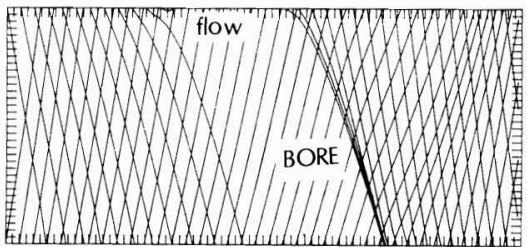

Fig. 6. Contours in the $(x, t)$ plane of model prediction for case $1(\varepsilon=0.8, \phi=0.25, \gamma=0.1)$ : (a) velocity; note the shorter, more intense flood currents; (b) water level; note the rapid change of water level from ebb to flood at any position; (c) characteristic curves; up- and downward sloping characteristics represent seaward and landward propagating long gravity waves, converging characteristics indicate the presence of a tidal bore.

speed of about $3 U$. Prior to flood, the flow at the mouth of the estuary is supercritical, i.e., particle velocities are greater than the phase velocity of the tidal wave. Thus, no upstream propagating characteristics enter from the mouth for almost $T / 6$, as they are all swept downstream relative to the channel.

Fig. 7 shows the momentum balance at three grid points for a single tidal cycle. Close to the mouth $(x=0.09)$ the acceleration term, $\partial_{t} u$, contributes only near slack water and is negligible most of the time. Furthermore, its average over one cycle $T$ is zero. The dominant momentum balance there is between the friction, the advection $\left(\frac{1}{2} \partial_{x} u^{2}\right)$, and the slope $\left(\partial_{x} \eta\right)$ terms. No tidal bore passes $x=0.09$. However, the free surface slope changes within $T / 12$ from its ebb to maximum flood value. Bores do pass both $x=0.35$ and 0.65 . All terms contribute then, but friction diminishes 
(a)

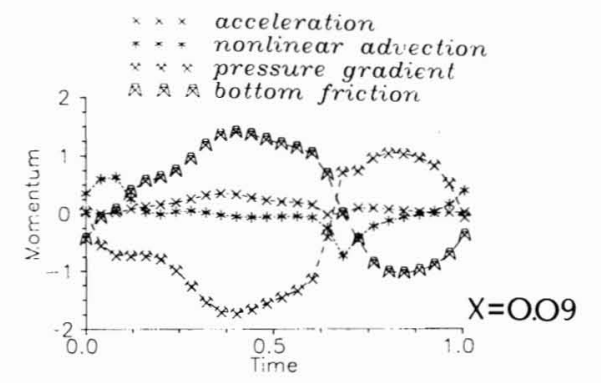

(b)

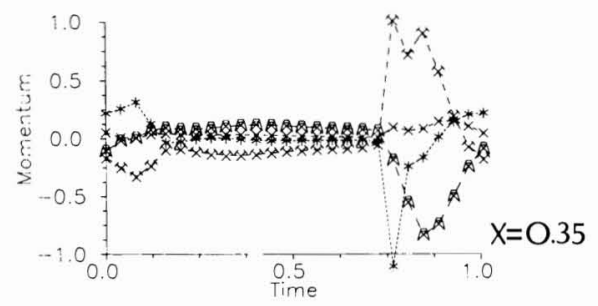

(C)

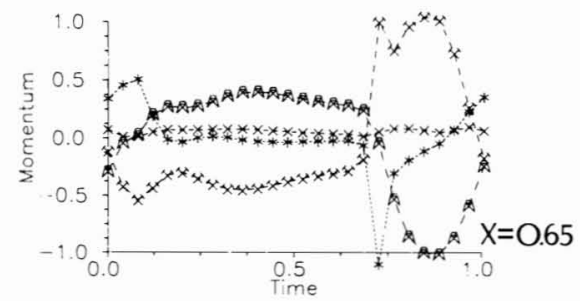

Fig. 7. Model momentum balance for a single tidal cycle of case $1(\varepsilon=0.8, \phi=0.25, \gamma=0.1)$ at three locations: (a) the bore (see Fig. 6c) is weak near the mouth where nonlinear advection and friction balance the free surface slope; further landward ( $b$ and $c$ ) the slope term changes sign quickly at about $t=0.7$, a bore passes then. Note the diminishing importance of the frictional term with landward position from (a) to (c). During the bore's passage friction and acceleration balance the slope and nonlinear advection term.

upstream. The balance at $x=0.65$ clearly shows that the bore's passage rapidly alters the momentum balance; otherwise all terms are small and nearly constant.

Case 2 represents a short, frictionally dominated estuary with $\varepsilon=0.8, \phi=0.1$, and $\gamma=0.1$. Fig. 8 depicts model predictions of velocity, water level, and characteristics. Note that the -0.25 contour line does not advance far into the estuary. The nearly straight characteristics in Fig. 8c indicate that no tidal bore forms. The momentum balance (not shown) resembles that of the Conwy Estuary where the major balance was between the water surface slope and friction (hydraulic balance).
The velocity and water level contours in Fig. 6 (case 1) and Fig. 8 (case 2) appear qualitatively similar, but exhibit two important differences.

(a) The velocities are higher and change more rapidly in time and space for lower friction (case 1); hence, nonlinear advection is more important in the momentum balance of case 1 when compared with case 2 .

(b) A time averaged slope present in case 2 is weak in case 1 .

Tidal bores tend to form in estuaries where the frictional bottom stresses are relatively small. The two cases just discussed support this argument, since for high friction (case 2) no bore develops, but for low friction (case 1) a bore forms. Prandle (1985) supports this finding: he modeled the linear tidal response of the Bristol Channel, the Bay of Fundy, and 8 other estuaries. For the former two estuaries, where bores develop frequently, he used

(a)

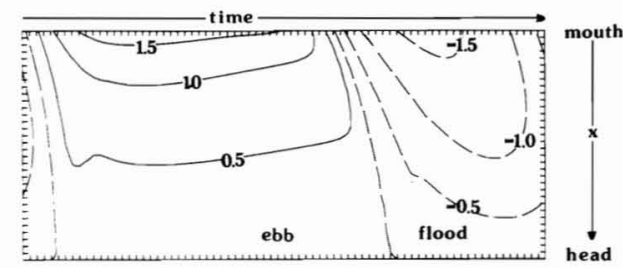

(b)

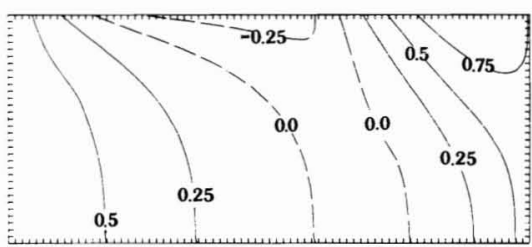

(C)

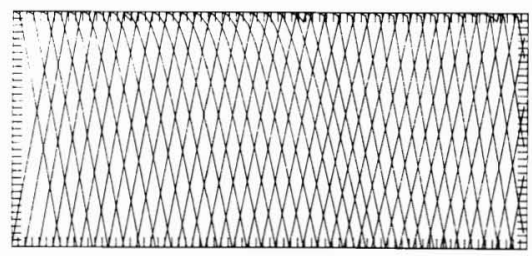

Fig. 8. As Fig. 6, but for case $2(\varepsilon=0.8, \phi=0.1, \gamma=0.1)$. Shown are (a) velocity, (b) water level, and (c) characteristic curves. As in Fig. 6, the flood currents are short in duration and high in magnitude. The negative water level contours do not advance far into the channel, therefore causing a strong time averaged free surface slope which is typical for hydraulic regimes. No bore is present, as the almost straight characteristics indicate. 
a frictional coefficient which was an order of magnitude smaller than the coefficients in his other 8 estuaries. However, his model does not account for nonlinearities and does not model bores.

\subsection{Moderately nonlinear flows: $\varepsilon=0.4$}

Cases 3 to 7 are representative examples of moderately nonlinear flows $\left(\varepsilon \equiv \eta_{0} / H=0.4\right)$. We vary the frictional parameter $\phi=L_{\mathrm{f}} / L_{\lambda}$ first for geometrically short estuaries $\left(\gamma=L_{\mathrm{g}} / L_{\lambda}=0.1\right.$, cases 3-5) and then for geometrically longer estuaries $(\gamma=0.5$, cases 6 and 7).

(a)
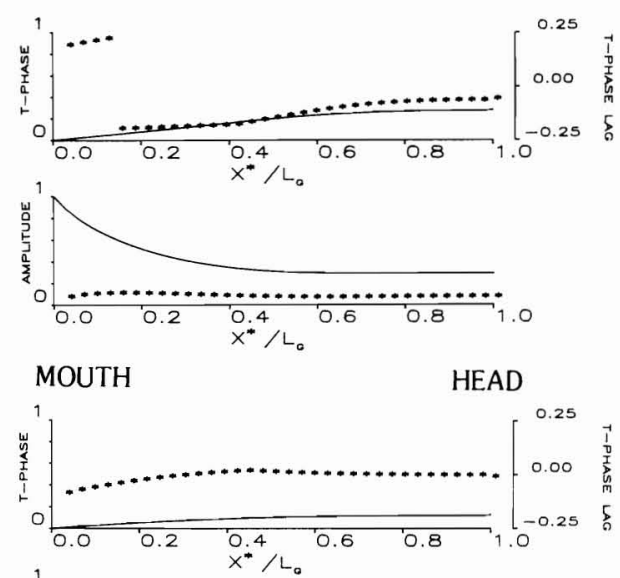

(b)

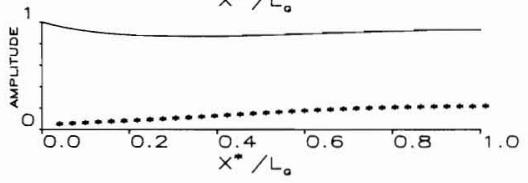

(C)
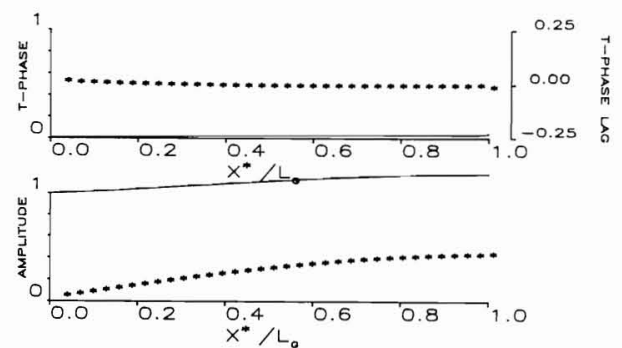

Fig. 9. Amplitude and phase propagation into the estuary of the forcing frequency constituent ( $T$-tide, solid line) and its first harmonic ( $T / 2$-tide, symbols): (a) case 3 $(\varepsilon=0.4, \phi=0.1, \gamma=0.1)$; (b) case $4 \quad(\varepsilon=0.4, \phi=0.25$, $\gamma=0.1)$; and (c) case $5(\varepsilon=0.4, \phi=0.5, \gamma=0.1)$. No bore develops in any of the cases. The amplitude of the $T$-tide increases with increasing $\phi$ (decreasing friction). As friction decreases from (a) to (c) the fundamental constituent and its first harmonic become phase locked.
A common effect of nonlinearity in tidal flows is the production of temporal variations that represent harmonics of the basic forcing period $T$ (Aubrey and Speer, 1985). Thus, we expect our model results to contain Fourier constituents of period $T / 2, T / 3$, etc. The amplitude distribution with $x$ of the first harmonic constituent serves as an indicator of the strength of nonlinearity.

Fig. 9 depicts the results of a harmonic analysis of water level for the forcing period $T$ and its first harmonic of period $T / 2$ for short estuaries $(\gamma=0.1)$. It shows the along channel phase and amplitude variation for cases 3,4 , and 5 . Friction decreases successively as we increase $\phi$ from 0.1 to 0.5 . Solid lines represent the results for the $T$ tide, whereas the dots represent those for the first harmonic. The phase of the $T / 2$ tide plotted is the local phase difference $\theta \equiv \theta_{T}-2 \theta_{T / 2}$ between the two periods analyzed, where $\theta_{T}$ and $\theta_{T / 2}$ are the respective phases of the $T$ - and $T / 2$ tide. For case 3 (Fig. 9a) friction quickly damps the amplitude of the $T$ tide and keeps that of the $T / 2$ tide small. For

(a)
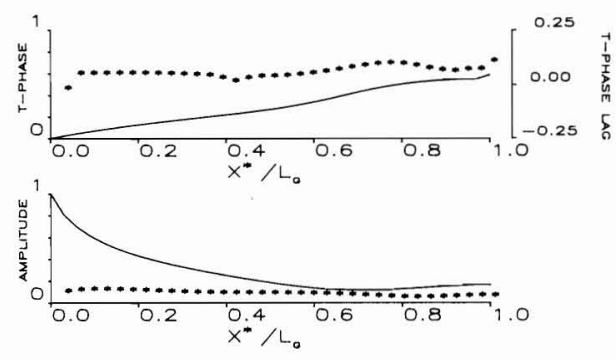

MOUTH

HEAD

(b)
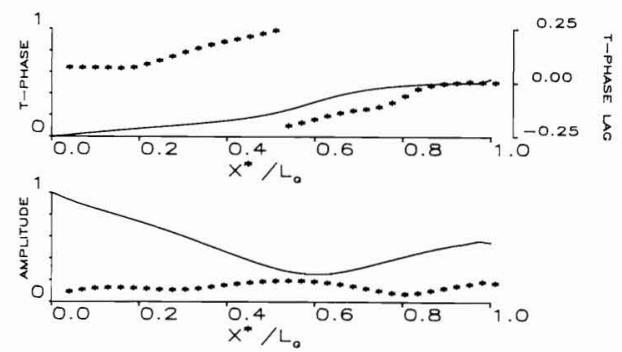

Fig. 10. As Fig. 9 but for: (a) case $6(\varepsilon=0.4, \phi=0.25$, $\gamma=0.5)$ and (b) case $7(\varepsilon=0.4, \phi=0.5, \gamma=0.5)$. Bores develop in both cases since the characteristic curves (not shown) coalesce. The amplitude of the $T$-tide decreases in the middle part of the estuary and then increases in the upper parts. The phase difference between the $T$ - and the $T / 2$-tides varies with position; therefore the two tides are not phase locked. 
$x^{*} / L_{\mathrm{g}}>0.4$ the $T / 2$ tide is phase locked to the $T$ tide, i.e. $\theta$ is nearly constant. The amplitude of the $T / 2$ tide increases greatly with upstream position in cases 4 and 5. However, the characteristics (not shown) do not coalesce and therefore no bores develop.

Finally, we discuss a similar set of experiments for long estuaries, i.e., $\gamma=0.5$. Weak or marginal bore development occurs in case $6(\phi=0.25)$ and case $7(\phi=0.5)$ for which Fig. 10 depicts the amplitude and phase variations along the channel. For both cases, the amplitudes of the $T$ tide at $x^{*} / L_{\mathrm{g}}=0.6$ reduce to less than $30 \%$ of their value at the mouth. The amplitude of the $T / 2$ tide increases and reaches a maximum where the $T$ tide has its minimum. The characteristic curves (not shown) coalesce in both cases, hence bore development is evident for cases 6,7 .

\section{Summary}

Observations and modeling of barotropic estuarine tidal dynamics have shown that estuaries are not necessarily simple co-oscillations of the coastal oceans. During the last three decades much has been learnt about the physical mechanisms causing such nonlinear tidal motion (Kreiss, 1957; Le Blond, 1978; Aubrey and Speer, 1985) and tidal wave breaking (Abbott, 1956). In this study we have demonstrated that the tidal wave entering estuaries may undergo drastic distortions. We have also presented a unifying model of barotropic tides in estuaries, have defined the dimensionless parameters that govern them, and have modeled them numerically.

A scaling analysis of the governing equations revealed that the following parameters govern barotropic tidal dynamics in estuaries:

(1) $\varepsilon \equiv \eta_{0} / H$, the ratio between the tidal amplitude at the estuarine mouth and the mean depth in the estuary. This parameter indicates the nonlinearity of the estuary (Ianniello, 1977);

(2) $\phi \equiv L_{\mathrm{f}} / L_{\lambda}$, the ratio between the frictional and the tidal length scales;

(3) $\gamma \equiv L_{\mathrm{g}} / L_{\lambda}$, the ratio between the geometric and the tidal length scales.

The last two parameters determine which term balances the free surface slope in the momentum balance.
In Fig. 11 we sketch the domain of different estuarine regimes in the three dimensional parameter space $(\varepsilon, \phi, \gamma)$. It shows in particular the $(\varepsilon, \phi, \gamma)$ co-ordinates for the seven cases studied in Section 5. We draw three different planes of $\varepsilon$ corresponding to nearly linear $(\varepsilon=0.1)$, moderately nonlinear $(\varepsilon=0.4)$, and highly nonlinear $(\varepsilon=0.8)$ estuarine flows. We also sketch two surfaces marking the tentative boundaries between three dynamic regimes: damped tidal waves, hydraulic balances, and bores. Though approximate, these boundaries are based on the seven cases discussed as well as on many more not presented. By estimating the parameters $(\varepsilon, \phi, \gamma)$ for a particular estuary one may thus predict the dynamic regime of that estuary in advance.

The lowest $\varepsilon$ level $(\varepsilon=0.1)$ and below is the domain of linear and weakly nonlinear tidal waves (Ianniello, 1977; Prandle, 1985). The symbol $L$ on this plane indicates the position of the linear test case used for model verification (Muenchow, 1988). Only for large friction $(\phi \ll 1)$ do we expect hydraulic responses here. The $\varepsilon=0.4$ level exhibits a variety of regimes for different length scale ratios $\phi$ and $\gamma$. For large friction $(\phi<0.1)$, we find hydraulic balances, while tidal bores are possible for $\phi$ and $\gamma$ both larger than 0.5. The domain between these regimes for $\varepsilon=0.4$ consists of nonlinearily distorted, but damped waves. Finally, for strongly nonlinear tidal responses $(\varepsilon=0.8)$, either

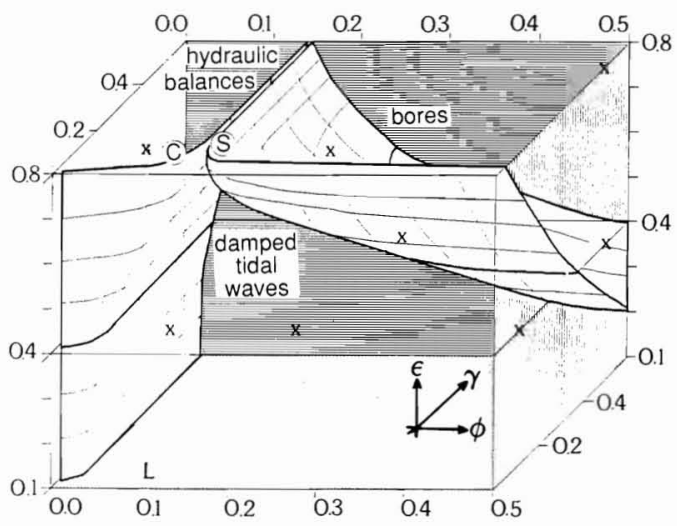

Fig. 11. Sketch of the dynamic regimes in parameter space $(\varepsilon, \phi, \gamma)$. Each point in $(\varepsilon, \phi, \gamma)$ space represents an estuary, i.e., $\mathrm{S}$ and $\mathrm{C}$ indicate the position of the Severn and Conwy estuaries, $\mathrm{L}$ the linear test case, and $x$ indicates the position of the eight numerical cases summarized in Table 1. 
hydraulic balances or bores dominate the major part of the $(\phi, \gamma)$ plane. The Conwy Estuary $(\varepsilon=0.8, \phi=0.11, \gamma=0.07$; Section 4) and the Severn Estuary $(\varepsilon=0.8, \quad \phi=0.15, \quad \gamma=0.14$; Muenchow, 1988) represent qualitatively different model responses, since only for the Severn model do bores develop. In contrast, the Conwy Estuary model displays hydraulic balance. The same distinction is found in observations of the two estuaries (Rowbotham, 1983).

\section{Acknowledgements}

The convenient access to data from the Welsh Water Authority (Dr. Ackroyd) and Birmingham
University (Dr. West) is greatfully acknowledged. Also, we wish to thank one of the anonymous reviewers for correcting a serious error in our model formulation.

The senior author (AM) undertook the reported field work while participating in an MSc course at the School of Ocean Sciences at the University College of North Wales (UCNW). There, Dr. J. H. Simpson generously overcame many hindrances and provided initial support without which this work would not have been possible. While there, one of us (AM) was supported by the West-German Government (BAFÖG). At the University of Delaware, the National Science Foundation funded the work through grant OCE-8711299.

\section{REFERENCES}

Abbott, M. 1956. A theory of the propagation of bores in channels and rivers. Proc. Cambridge Phil. Soc. 52, 344-362.

Aubrey, D. G. and Speer, P. E. 1985. A study of nonlinear tidal propagation in shallow inlet/estuarine systems; part I: observations. Estuar. Coast. Shelf Sci. 21, 185-205.

Cornish, V. 1934. Ocean waves and kindred physical phenomena. Cambridge, U.K.: University Press.

Hansen, W. 1956. Theorie zur Errechnung des Wasserstandes und der Strömungen in Randmeeren nebst Anwendungen. (In German) Tellus 8, 287-300.

Houghton, D. D. and Kasahara, A. 1968. Non-linear shallow fluid over an isolated ridge. Comm. Pure Appl. Math. 13, 1-13.

Ianniello, J. P. 1977. Tidally induced residual currents in estuaries of constant depth and breadth. J. Mar. Res. 35, 755-786.

Kreiss, H. 1957. Some remarks about nonlinear oscillations in tidal channels. Tellus 9, 53-68.

Lax, P. D. and Wendroff, B. 1960. Systems of conservation laws. Comm. Pure Appl. Math. 13, 213-237.

LeBlond, P. H. 1978. On tidal propagation in shallow rivers. J. Geophys. Res. 83, 4717-4721.

Mazumder, N. C., Chaterjee, P. K. and Basak, S. K. 1984. Generation of a bore. J. Waterw., Port, Coast., Ocean Engineer. 110, 159-170.

Muenchow, A. 1988. Nonlinear tides and bores in shallow. estuaries. MS thesis, University of Delaware, Newark, DE, USA.
O'Donnell, J. and Garvine, R. W. 1983. A time dependent 2-layer model of buoyant plume dynamics. Tellus $35 \mathrm{~A}$, 73-80.

Prandle, D. 1985. Classification of tidal response in estuaries from channel geometry. Geophys. J. R. Astr. Soc. 80, 209-221.

Richtmayer, R. D. and Morton, K. W. 1967. Difference methods for initial value problems. New York, N.Y.: Interscience.

Rowbotham, F. 1983. The Severn bore, 3rd edition. North Pomfret, Ve: David's and Charles.

Shiono, K. and West, J. R. 1987. Turbulent perturbations of velocity in the Conwy Estuary. Estuar. Coast. Shelf Sci. 25, 533-553.

Speer, P. E. and Aubrey, D. G. 1985. A study of nonlinear tidal propagation in shallow inlet/estuarine systems, part II: theory. Estuar. Coast. Shelf Sci. 21, 207-222.

Stoker, J. J. 1947. The formation of breakers and bores. Comm. Pure Appl. Math. 1, 1-87.

Wallis, S. G. and Knight, D. W. 1984. Calibration studies concerning a one-dimensional numerical tidal model with particular reference to resistance coefficients. Estuar. Coast. Shelf Sci. 19, 541-562.

West, K. and Mangat, J. S. 1986. The determination and prediction of longitudinal dispersion coefficients in a narrow, shallow estuary. Estuar. Coast. Shelf Sci. 22, 161-181. 